Cell Research (1999), 9, 243-253

\title{
The convergent point of the endocytic and autophagic pathways in leydig cells
}

\author{
Yi Jing, Xue Ming Tang* \\ Department of Biophysics and Cell Biology, Shanghai Se- \\ cond Medical University, Shanghai 200025, China
}

\begin{abstract}
Endocytic tracers and marker enzyme of lysosomes were used in the present study to analyze the processes of autophagocytosis and endocytosis, and the convergent point of these two pathways in Leydig cells. The endocytic and autophagic compartments can be easily identified in Leydig cells, which makes easier to define the stages of two pathways than was possible before. The evidences indicated that the late endosomes (dense MVBs) deliver their endocytosed gold tracers together with lysosomal enzymes to the early autophagosomes and they are the convergent point of the two pathways. During this convergent process, the early autophagosomes transform into late autophagosomes and the late endosomes transform into mature lysosomes.
\end{abstract}

Key words: Autophagocytosis, endocytosis, endosomes, lysosomes.

\section{INTRODUCTION}

Endocytosis and autophagocytosis (autophagy) are the major pathways for materials to be transported to lysosomes in animal cells, the former being responsible for uptake of extracellular constituents and the latter for degradation of cytoplasmic constituents.

The endocytic pathway can be visualized in the electron microscope by adding a readily detectable tracer to the extracellular medium and leaving the cells for varying lengths of time to take it up by endocytosis. The distribution of the lysosomally targeted ligands

\footnotetext{
* Corresponding author: Xue Ming TANG, Department of Biophysics and Cell Biology, Shanghai Second Medical University, 280 South Chongqing Road, Shanghai 200050, China
} 
Convergent point of the endocytic and autophagic pathways

after their uptake reveals that internalized tracers first appear in early endosomes at the cell periphery and then in late endosomes in the perinuclear region, before they are observed in lysosomes[1]. However, uncertainty remained on the structure and function of early and late endosomes.

Compared to the endocytic pathway, the autophagic pathway has been poorly characterized. The low frequency of autophagy makes it rather difficult to study the process and mechanism of autophagy in normal cells. So far, most of the information about autophagy came from induced autophagy[2-3]. During the past years, we tried to estimate the relative frequency of the autophagy in 32 cell types under normal condition. We found that the relative frequency of autophagy in Leydig cells is much higher than in other cell types in rats[4], and Leydig cell is a good model to study the process and mechanism of autophagy. In the present study, we use this cell model to investigate the process of endocytosis and autophagocytosis, and then to define the convergent point of endocytic and autophagic pathways in normal rat Leydig cells.

\section{MATERIALS AND METHODS}

\section{Morphology and cytochemistry}

Twelve adult male Wistar rats were used in the first part of this study. Each testis was fixed by perfusion through the abdominal aorta with $2 \%$ glutaraldehyde buffered in $0.1 \mathrm{M}$ sodium cacodylate buffer containing $0.05 \%$ calcium chloride $(\mathrm{pH} 7.4)$. The organs were removed after 5 min of perfusion, trimmed into $1 \times 1 \times 10 \mathrm{~mm}$ pieces and placed in the same fixative for $1 \mathrm{~h}$ at 4 ${ }^{\circ} \mathrm{C}$. The tissue was then washed overnight in $0.1 \mathrm{M}$ sodium cacodylate buffer containing $4 \%$ sucrose and kept in the same buffer at $4{ }^{\circ} \mathrm{C}$.

For cytochemical experiments, the tissue blocks from six animals were sectioned at $75 \mu \mathrm{m}$ with a Sorvall TC-2 tissue sectioner. For demonstration of activity of cytidine monophosphatase (CMPase), one of lysosomal marker-enzymes, the sections were incubated for $60 \mathrm{~min}$ at $37{ }^{\circ} \mathrm{C}$ in a medium containing $2 \mathrm{mM}$ cytidine monophosphate, $2 \mathrm{mM}$ cerium chloride and $0.1 \mathrm{M}$ sodium acetate buffer ( $\mathrm{pH}$ 5.0) with 5\% sucrose. Sections were incubated in a medium free from substrate served as controls. After incubation, the sections were postfixed in $1.5 \%$ ferrocyanide reduced osmium tetroxide and then dehydrated in ethanol, followed by infiltration in propylene oxide and embedding in Epon.

For morphological observation, the glutaraldehyde-perfused tissues were postfixed and processed as described above after an overnight wash in cacodylate buffer.

\section{Endocytic tracers}

Two type of lectins, concanavalin A (ConA) and wheat germ agglutinin (WGA), were used as tracing reagents for adhesive endocytosis, and a ligand specific to LH receptor on Leydig cell membrane, human choriogonadotropin (hCG), was used for receptor-mediated endocytosis. They were labeled with $15 \mathrm{~nm}$ colloidal gold and in concentrations of $0.6,0.5$ and $0.1 \mathrm{mg} / \mathrm{ml}$ respectively. Thirty-six rats were divided into three groups. Each rat was administered with ConA-, WGA- or hCG-gold, according to their grouping, by injecting to testis interstitially at a dose of $0.1 \mathrm{ml}$. Two rats of each group were sacrificed at each time interval, i.e. at 5, 15, 30 min and 1, 2, 3 and $6 \mathrm{~h}$ after a single injection, and the testicular tissues were fixed and embedded using the methods described above. 


\section{RESULTS}

\section{Morphology and cytochemistry of autophagic activity}

Leydig cells of normal adult rats showed an abundance of interconnected cisternae of smooth and rough endoplasmic reticulum (ER) as well as mitochondria and peroxisomes. They also showed a large Golgi region and a variable number of lysosomes and multivesicular bodies (MVBs). Autophagosomes, variable in content, were frequently observed in electron micrographs of Leydig cells (Fig 1). Early signs of autophagocytosis appeared in the form of flattened membranous profiles, a C-shaped configuration, showing two distinct membranes delimiting a narrow lumen that was narrower than that of ER cisternae and Golgi saccules. Being referred to as preautophagosomes, the C-shaped double membrane structure extended to demarcate a cytoplasmic territory containing normal-looking mitochondria and smooth ER cisternae (Fig 2a). The double membrane profile also completely enclosed small cytoplasmic territories. In the latter structures, referred to as early autophagosomes, recognizable organelles such as mitochondria and smooth ER were present (Fig 2b). In the subsequent course of their evolution,

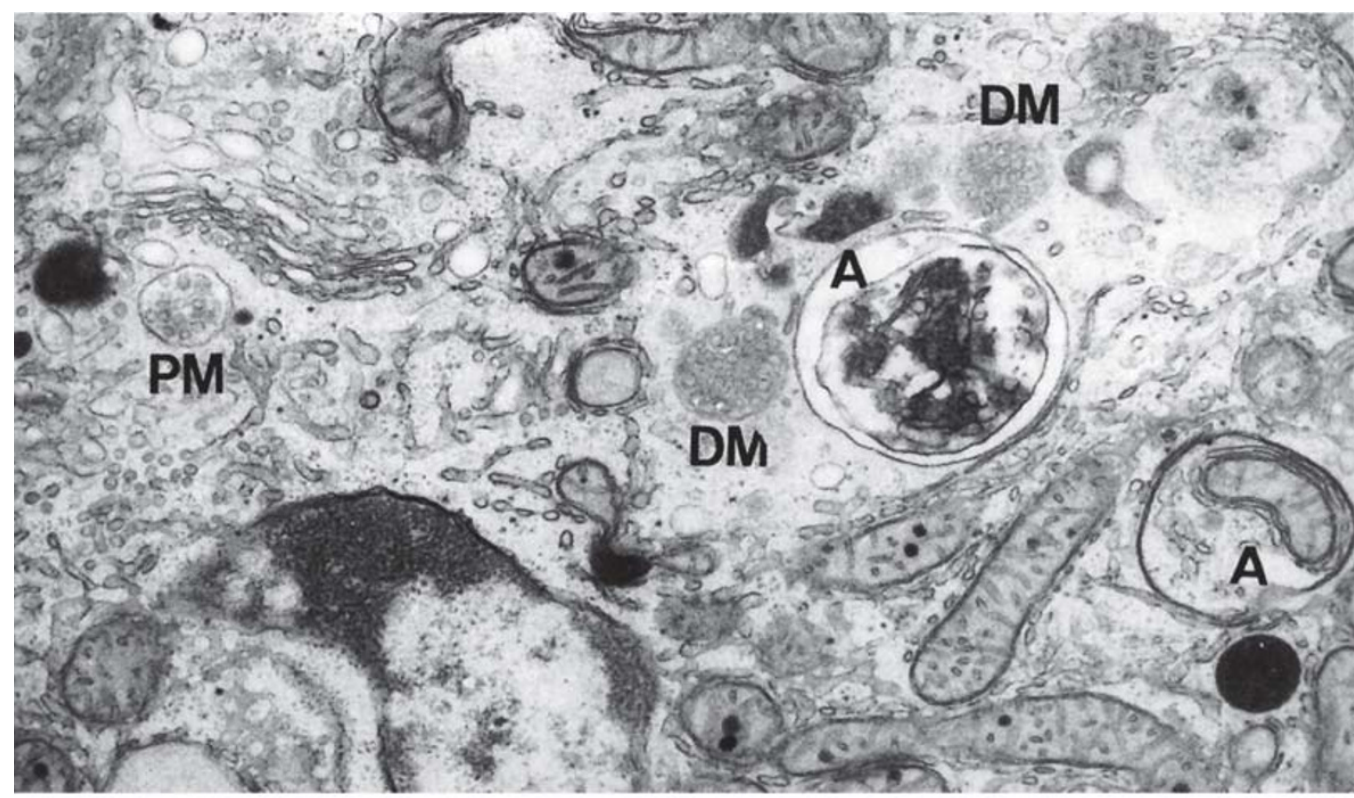

Fig 1.

Electron micrograph showing a small portion of a rat Leydig cell. In addition to smooth endoplasmic reticulum and mitochondria, this field shows a pale MVB $(\mathrm{PM})$, two dense MVBs (DM) and two autophagosomes (A), $35000 \times$. 
Convergent point of the endocytic and autophagic pathways

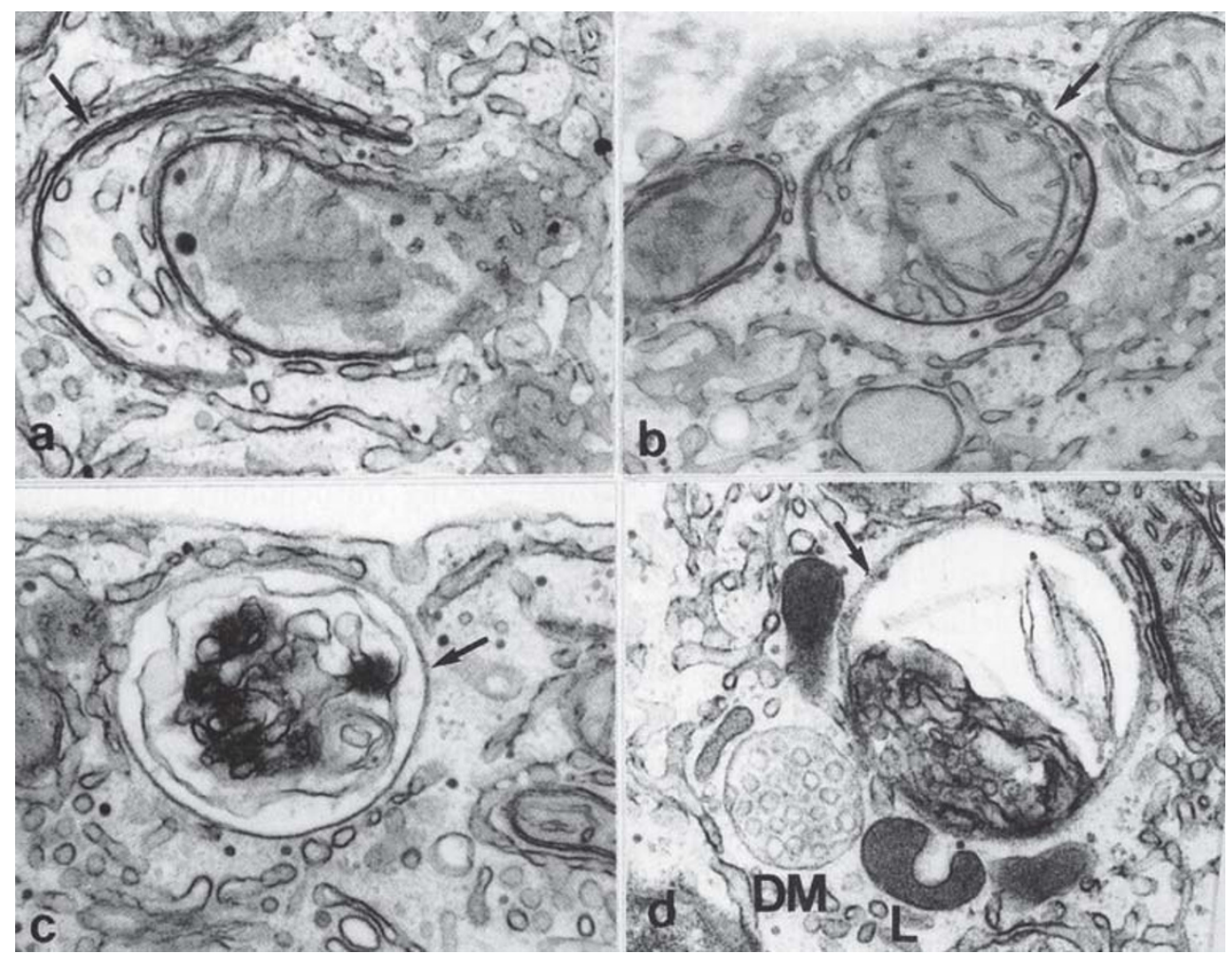

Fig 2.

Photographs showing the images of different stages of autophagocytosis: (a) preautophagosome, $50000 \times$; (b) early autophagosome, $40000 \times$; and (c) late autophagosome, $40000 \times$; (d) a late autophagosome (arrow) shows a close relation with a dense MVB (DM) and lysosomes (L), $40000 \times$.

autophagosomes seemingly transformed to take on a wide variety of appearances, such elements were referred to as late autophagosomes(Fig 2c). Of these elements, some presented a large space between two delimiting membranes and enclosed organelles that were occasionally but not always recognizable. Others showed a single delimiting membrane and a granulated and membranous content, the latter being of unrecognizable origin. Late autophagosomes often showed a very close proximity to MVBs and lysosomes (Fig 2d). Although rare, images of exocytosis of the content of late autophagosomes were observed, and cellular debris in the form of concentric membranous profiles was commonly seen in the spaces between Leydig cells.

In sections treated to show CMPase activity, lysosomes and a network of membranous tubules seen on the trans region of the Golgi apparatus contained CMPase reaction products. The CMPase reactivity of autophagosomes varied depending on the stage of evolution. The flattened preautophagosomal membranous profiles were unreactive for CMPase as were the early autophagosomes showing two membranes separated by a narrow 
lumen. The late autophagosomes delimited by a single membrane and containing granular or membranous material of unrecognizable source showed CMPase activity. Not infrequently, these late autophagosomes were closely apposed to strongly reactive MVBs and lysosomes (Fig 3a). Late autophagosomes being exocytosedstill showed some CMPase activity (Fig 3b).

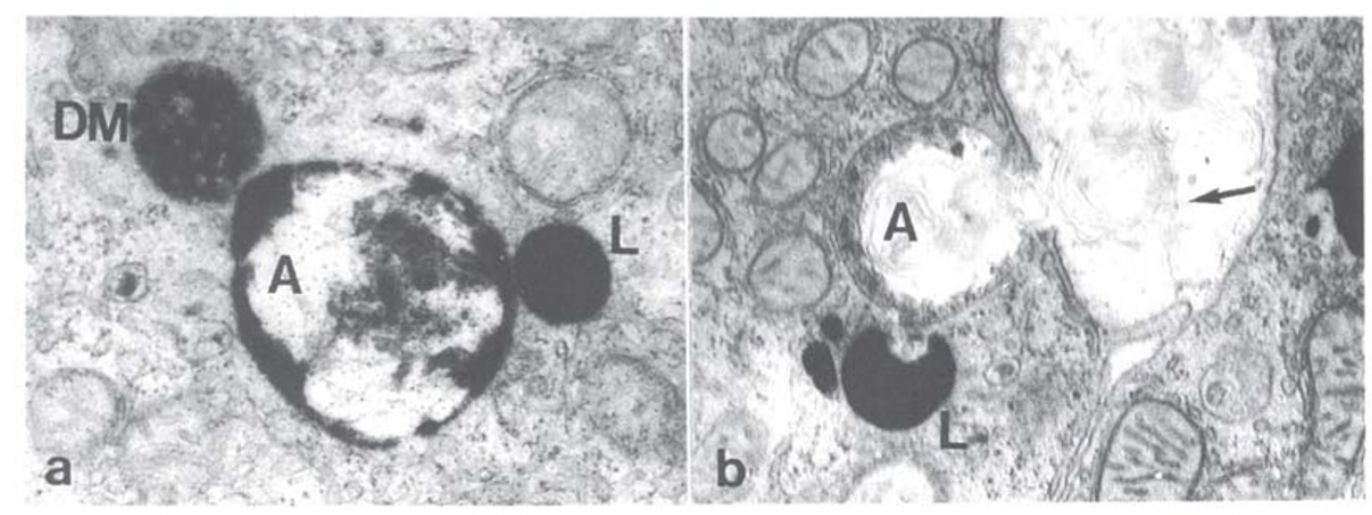

Fig 3.

Photographs from tissues treated to show CMPase activity: (a) A dense MVB (DM) and a lysosome (L) show a very close association with a autophagosome (A). All these structures are positive for CMPase, $40000 \times$; (b) A lysosome (L) showing strong CMPase reaction is adjacent to the exocytosing late autophagosome (A), the intercellular space contains membranous material (arrow) that appears to be exocytic in origin, $25000 \times$.

\section{Tracer studies of endocytic activity}

Tracer experiment showed that ConA-gold, WGA-gold and hCG-gold took the same lysosomal pathway of endocytosis in Leydig cells. They bound to their binding sites (for ConA-gold and WGA-gold) or receptors (for hCG-gold) at the cell surface and rapidly internalized into small endocytic vesicles at 5 min after injection (Fig $4 \mathrm{a}, \mathrm{b}$ ). The tracers were next found in small pale MVBs at $15 \mathrm{~min}$ (Fig 4c) and in large pale MVBs at $30 \mathrm{~min}$ after injection. The pale MVBs were characterized by their pale matrix and paucity of internal vesicles. The small pale MVBs often had an irregular shape with an extension of membranous tubule, whereas the large pale MVBs usually had a spherical shape. At $1 \mathrm{~h}$ after injection, dense MVBs contained tracers, but most of the lysosomes were unlabeled (Fig 4d). The dense MVBs were characterized by their dense matrix and filling with internal vesicles. Most of the lysosomes became labeled at the $2 \mathrm{~h}$ and later time intervals. The dense MVBs and lysosomes often showed a very close association with autophagosomes that also became labeled with tracers at late time intervals (Fig 4e). At early time intervals, however, autophagosomes were never tracer-contained, despite there were occasionally some labeled pale or dense MVBs close to them (Fig 4f). 


\section{Convergent point of the endocytic and autophagic pathways}

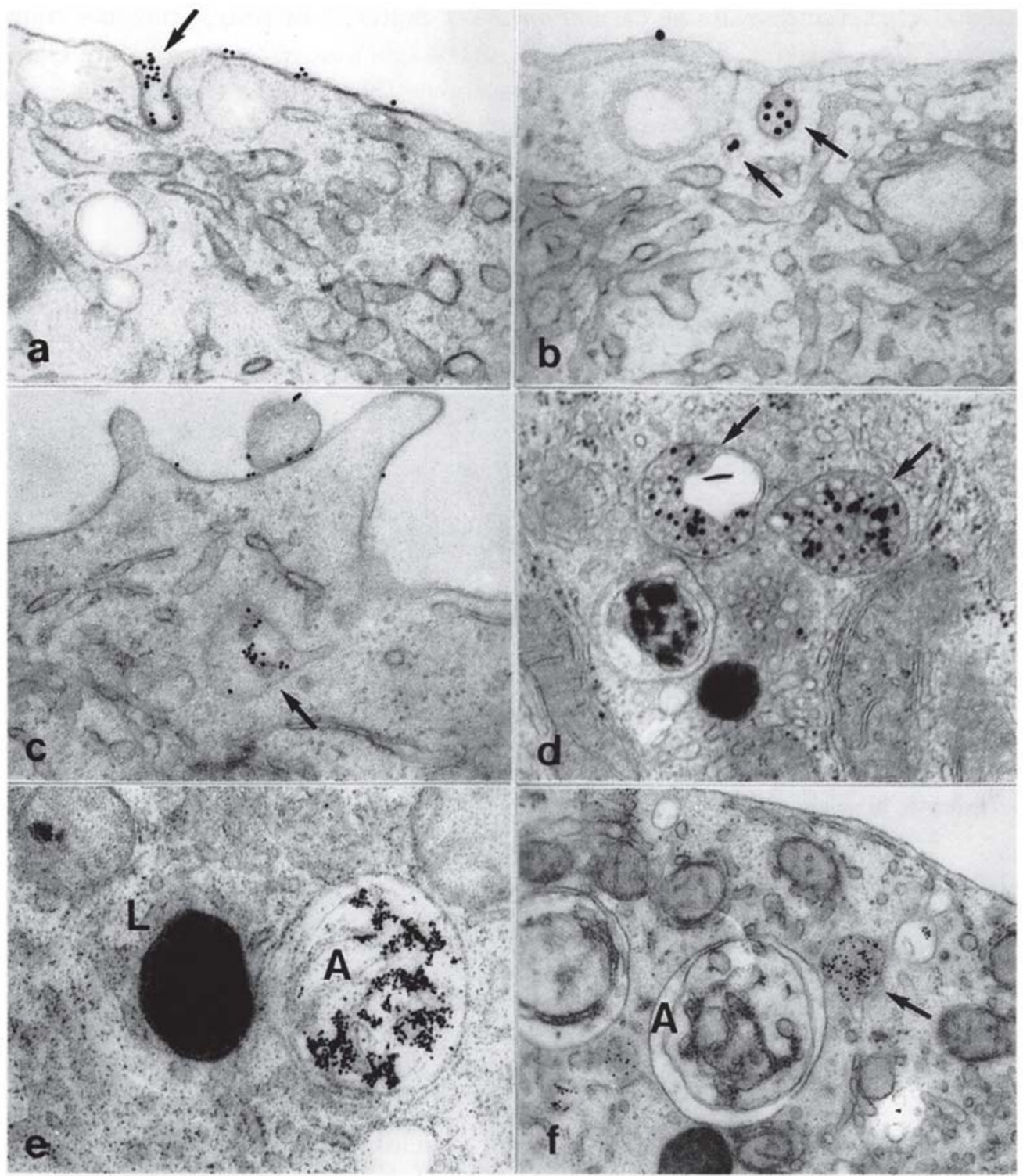

Fig 4.

Photographs of Leydig cells after injection of tracers: (a) At 5 min after injection of WGA-gold, the gold particles are seen bound to the cell surface and endocytic pit (arrow), $50000 \times$; (b) At 5 min after injection of ConA-gold, the gold particles appear in the endocytic vesicles (arrow), $60000 \times$; (c) At 15 min after injection of ConA-gold, the tracer appears in the small pale MVB (arrow), $40000 \times$; (d) At 1 $\mathrm{h}$ after injection of ConA-gold, the dense MVBs (arrow) are seen to contain tracer, $50000 \times$; (e) At $2 \mathrm{~h}$ after injection of ConA, the lysosome (L) become labeled with tracer, it shows a very close association with the autophagosome (A) which also contains gold particles, $35000 \times$; (f) At 15 minutes after injection of WGA-gold, the gold particles are seen in the small MVB (arrow), but the adjacent autophagosome (A) is not labeled with tracer, $25000 \times$. 
Cytochemical experiment showed that, in contrast to lysosomes which were CMPase positive, the small and large pale MVBs were CMPase negative (Fig 5a). The dense MVBs always showed CMPase reactivity, though varying in intensity (Fig 5b). Some dense MVBs were slightly reactive, and others were strongly reactive. The late autophagosomes, similarly to the dense MVBs and lysosomes, showed strong CMPase reactivity.

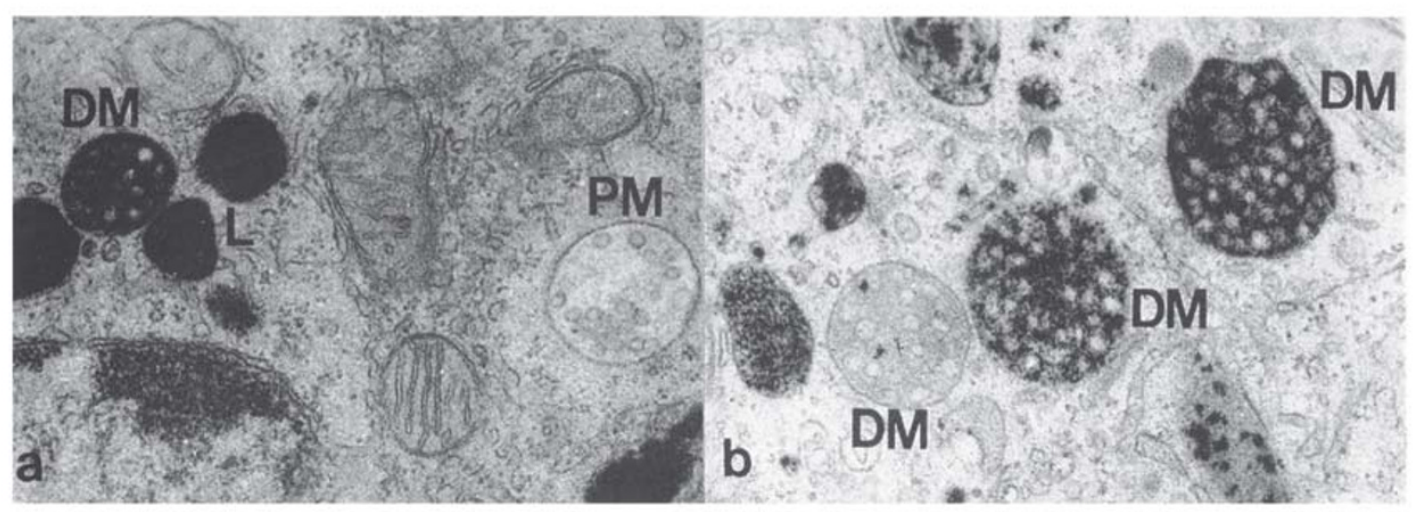

Fig 5.

Photographs of Leydig cells showing CMPase reactivity: (a) The dense MVB (DM) and lysosomes (L) are strongly reactive with the CMPase substrate, but the pale MVB (PM) is CMPase negative, $40000 \times$; (b) The dense MVBs $(\mathrm{DM})$ show CMPase reactivity in different degree, $50000 \times$.

\section{DISCUSSION}

\section{The autophagic pathway in leydig cells}

Autophagy is a cellular process in which small portions of cytoplasm are segregated and destroyed by lysosomal enzymes. Our previous studies found that autophagy took place more frequently in Leydig cells than in any other cell types in rat[5-6]. In the present study, we take advantage of this cell model to delineate a more detailed process of autophagy occurred in fully differentiated cells.

In the present investigation, the early signs of autophagy, called preautophagosomes, can be easily seen in electron micrographs of Leydig cells, while they are rarely observed in other normal cell types and in cells where autophagy is induced. When the double membrane profile completely enclosed small cytoplasmic territories containing mitochondria and smooth ER, the early autophagosomes are formed. The transformation of an early autophagosome into a late one is accompanied by the loss of one of the two delimiting membranes, the partial disintegration of the enclosed content and the simultaneous 
Convergent point of the endocytic and autophagic pathways

acquisition of acid phosphatase activity. Enzymatic reactivity is acquired following a close association with CMPase-positive MVBs and lysosomes. After lysosomal degradation, the late autophagosomes become residual bodies. The residual material appears to be exocytosed as indicated by images of exocytosis and the accumulation of membranous debris in the spaces between Leydig cells. Overall, the abundance of diverse autophagosomal structures in Leydig cells allows outlining a process of autophagy in detail. For the functional implication of autophagy in these cells, our previous work suggest that autophagy takes part in regulation of steroid hormone secretion, which function as crinophagy does in the regulation of peptide hormone secretion[7].

\section{The endocytic pathway in leydig cells}

Many of the endocytosed materials end up in lysosomes, and this pathway was called lysosomal pathway of endocytosis. In this pathway, endosomes play a central role in directing the extracellular macromolecules internalized by endocytosis. In the lysosomal pathway of endocytosis, it is clear that internalized macromolecules first appear in early endosomes at the cell periphery and then in late endosomes in the perinuclear region, and finally the late endosomes are thought to convert into lysosomes. However, it is still not clear about the structure and function of early and late endosomes[8-10].

In the present study, endocytic tracers including ConA-gold, WGA-gold and hCG-gold and marker enzyme of lysosomal elements were used to analyze the early and late endosomes in Leydig cells. The results displayed that, in Leydig cells, these tracers all take the same itinerary destining to lysosomes, indicating that both adhesive-and receptor-mediated endocytosis have the same endocytic pathway. After binding to their binding sites or receptors at the cell surface, the tracers are internalized into small endocytic vesicles and then all found in the MVBs, first in pale MVBs that have irregular shape with tubular membranous extensions, subsequently in dense MVBs showing CMPase reactivity. This means that the endosomes in Leydig cells morphologically appear as MVBs. The pale MVBs function as early or sorting endosomes in which recycling receptors are sorted from lysosomal targeted ligands and the tubular regions are supposed to contain receptors destined to recycle. The dense MVBs work as late endosomes that appear incapable of fusing with newly formed endocytic vesicles, but accept transport vesicles bearing lysosomal enzymes from the Golgi apparatus and ultimately mature into lysosomes.

\section{The convergent point of endocytic and autophagic pathways}

The classical view is that the autophagosomes fuse with preexisting primary lysosomes to yield secondary lysosomes. That is, the autophagic and endocytic pathways converge at the lysosomal leve[11]. The later biochemical and cytochemical studies imply that the autophagic pathway may meet the endocytic pathway at an earlier, prelysosomal level [12]. The studies on the biogenesis of lysosomes have revealed that endosomes are also 


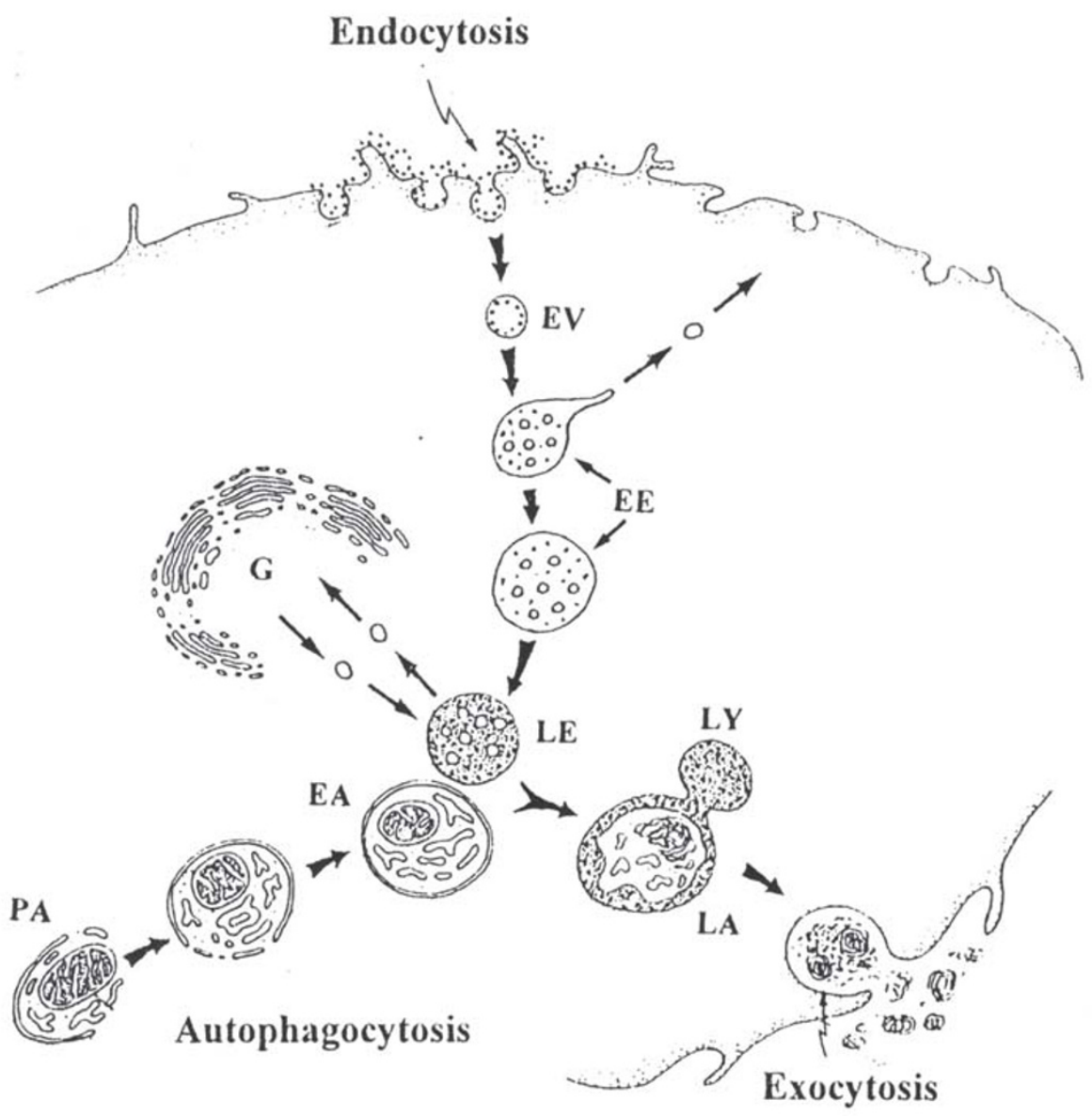

Fig 6.

Diagram showing the process and convergent point of endocytic and autophagic pathways in Leydig cell. In the endocytic pathway, the internalized tracers first appear in endocytic pit, endocytic vesicle (EV) and early endosome (EE), and then in the late endosome (LE) which accepts transport vesicles with lysosomal enzymes from the Golgi apparatus (G) and meets the autophagic pathway. In the autophagic pathway, the formation of autophagosome in Leydig cell can be divided into three steps: the preautophagosome (PA), the early autophagosome (EA) and the late autophagosome (LA). The late endosome and early autophagosome are the convergent point of the two pathways. During this convergent process, the early autophagosome transforms into late autophagosome and the late endosome transforms into mature lysosome (LY). After lysosomal degradation, the late autophagosome becomes residual, the residual material is eliminated by exocytosis.

endowed with lysosomal characteristics. Though it has been now accepted widely that the endosomes might be the meeting place of the autophagic and endocytic pathways[13], uncertainty remained on the precise site where the two pathways come together. Some 
Convergent point of the endocytic and autophagic pathways

authors indirectly suggested that the endocytic and autophagic pathways converge immediately after the formation of the first compartments, i.e., early endosomes and early autophagosomes of each pathway. Others believed that the convergence might be a multistage event[14-16]. In the present study, the endocytic and autophagic compartments can be readily identified in Leydig cells, which makes easier to define the stages of two pathways than was possible before. The close association between late endosomes (dense MVBs) and autophagosomes were considered to reflect fusion events. Two lines of evidence indicated that it is late endosomes (dense MVBs) which deliver their endocytosed gold tracers together with lysosomal enzymes to the early autophagosomes and where the two pathways converge (Fig 6). During this convergent process, the early autophagosomes transform into late autophagosomes and the late endosomes transform into mature lysosomes. First, no gold tracers can be seen in autophagosomes at early time intervals when early endosomes contain the tracers (before $1 \mathrm{~h}$ after injection), indicating that the CMPase-negative early endosomes do not deliver gold tracers to the autophagosomes. The two distinct pathways should converge at the later compartments rather than the initial or early ones. Secondly, the late endosomes contain endocytosed tracers as well as lysosomal enzymes. They often show very close association with autophagosomes that present the sequestered cytoplasmic constituents in varied degree of digestion, and these associate autophagosome also become labeled with tracers at late time intervals (later than $1 \mathrm{~h}$ after injection). It means the CMPase-positive late endosomes deliver both gold tracers and lysosomal enzymes to the early autophagosomes, and the hydrolytic digestion of the content of autophagosomes by lysosomal enzymes quickly makes them become late autophagosomes. This reveals the one-step convergence of endocytic and autophagic pathways, and explains why in electron micrographs, the autophagosomes with close association to the late endosomes are usually late autophagosomes.

\section{ACKNOWLEDGMENTS}

This project was supported by research grants from National Natural Science Foundation of China (No. 39670353).

\section{REFERENCES}

[1] Dunn KW, Maxfield FR. Delivery of ligands from sorting endosomes to late endosomes occurs by maturation of sorting endosomes. J Cell Biol 1992; 117:301-10.

[2] Kovacs AL, Reith A, Seglen O. Accumulation of autophagosomes after inhibition of hepatocytic protein degradation by vinblastine, leupeptin of a lysosomotropic amine. Exp Cell Res 1982; 137:191-201.

[3] Dunn WA. Studies on the mechanisms of autophagy: formation of the autophagic vacuole (Two articles). J Cell Biol 1990; 110:1923, 1933, 1935-45.

[4] Tang XM, Zhang HX, Yi J. Leydig cells-a normal cell model of cellular autophagy. Acta Biologiae Experimentalis Sinica. 1992; 25:39-47.

[5] Tang XM. The autophagic activity of Leydig cells in the normal rat testes. Acta Biologiae Experimentalis Sinica 1988; 21:119-29. 


\section{Yi J, XM Tang}

[6] Tang XM, Clermont Y, Hermo L. Origin and fate of autophagosomes in Leydig cells of normal adult rats. J Androl 1988; 9:284-93.

[7] Yi J, Tang XM. Functional implication of autophagy in steroid-secreting cells of the rat. Anat Rec 1995; 242:137-46.

[8] Rodman JS, Mercer RW, Stahl PD. Endocytosis and transcytosis. Curr Opin Cell Biol 1990; 2:664-72.

[9] Parton RG, Schrotz P, Bucci C, Gruenberg J. Plasticity of early endosomes. J Cell Sci 1992; 103:33548.

[10] Mayor S, Presley JF, Maxfield FR. Sorting of membrane components from endosomes and subsequent recycling to the cell surface occurs by a bulk flow process. J Cell Biol 1993; 1257-69.

[11] Hoyvik H, Gordon PB, Seglen PO. Convergence of autophagic and endocytic pathways at the level of the lysosome. Biochem Soc Trans 1987; 15:961-5.

[12] Lawrence BP, Brown WJ. Autophagic vacuoles rapidly fuse with preexisting lysosomes in cultured hepatocytes. J Cell Sci 1992; 102:515-26.

[13] Yokota S, Himeno M, Kato K. Formation of autophagosomes during degradation of excess peroxisomes induced by di-(2-ethylhexyl)-phthatate treatment. III. Fusion of early autophagosomes with lysosomal compartment. Eur J Cell Biol 1995; 66:15-24.

[14] Punnonen EL, Autio S, Kaija H, Reunanen H. Autophagic vacuoles fuse with the prelysosomal compartment in cultured rat fibroblasts. Eur J Cell Biol 1993; 61:54-66.

[15] Tooze J, Hollinshead M, Ludwig T, Howell K, Hoflack B, Kern H. In exocrine pancreas, the basolateral endocytic pathway converges with the autophagic pathway immediately after the early endosome. $\mathrm{J}$ Cell Biol 1990; 111:329-45.

[16] Liou W, Geuze HJ, Geelen JH and Slot JW. The autophagic and endocytic pathways converge at the nascent autophagic vacuoles. J Cell Biol 1997; 136:61-70.

Received Oct-14-1999. Revised Nov-1-1999. Accepted Nov-8-1999. 